\title{
Anatomical characteristics of the styloid process in internal carotid artery dissection: Case-control study
}

International Journal of Stroke 0(0) 1-6

(C) 2017 World Stroke Organization Reprints and permissions: sagepub.co.uk/journalsPermissions.nav DOI: $10.1177 / 1747493017730779$ journals.sagepub.com/home/wso (SAGE

\author{
José M Amorim', Daniela Pereira ${ }^{2}$, Marta G Rodrigues ${ }^{3}$, \\ José Beato-Coelho ${ }^{4}$, Margarida Lopes ${ }^{5}$, André Cunha ${ }^{3}$, \\ Sofia Figueiredo ${ }^{6}$, Mafalda Mendes-Pinto ${ }^{2}$, Carla Ferreira ${ }^{5}$, \\ João Sargento-Freitas ${ }^{4}$, Sérgio Castro ${ }^{3}$ and João Pinho ${ }^{5}$
}

\begin{abstract}
Introduction: Pathophysiology of cervical artery dissection is complex and poorly understood. In addition to well-known causative and predisposing factors, including major trauma and monogenic connective tissue disorders, morphological characteristics of the styloid process have been recently recognized as a possible risk factor for cervical internal carotid artery dissection.
\end{abstract}

Aims: To study the association of the anatomical characteristics of styloid process with internal carotid artery dissection.

Methods: Retrospective, multicenter, case-control study of patients with internal carotid artery dissection and age- and sex-matched controls. Consecutive patients with internal carotid artery dissection and controls with ischemic stroke or transient ischemic attack of any etiology excluding internal carotid artery dissection, who had performed computed tomography angiography, diagnosed between January 2010 and September 2016. Two independent observers measured styloid process length and styloid process distance to internal carotid artery.

Results: Sixty-two patients with internal carotid artery dissection and 70 controls were included. Interobserver agreement was good for styloid process length and styloid process-internal carotid artery distance (interclass correlation coefficient $=0.89$ and 0.76 , respectively). Styloid process ipsilateral to dissection was longer than left and right styloid process in controls $(35.8 \pm 14.4 \mathrm{~mm}$ versus $30.4 \pm 8.9 \mathrm{~mm}$ and $30.3 \pm 8.2 \mathrm{~mm}, \mathrm{p}=0.01 \mathrm{I}$ and $\mathrm{p}=0.008$, respectively). Styloid process-internal carotid artery distance ipsilateral to dissection was shorter than left and right distance in controls $(6.3 \pm 1.9 \mathrm{~mm}$ versus $7.2 \pm 2.1 \mathrm{~mm}$ and $7.0 \pm 2.3 \mathrm{~mm}, \mathrm{p}=0.003$ and $\mathrm{p}=0.026$, respectively). Internal carotid artery dissection was associated with styloid process length (odds ratio $=1.04 \mathrm{~mm}^{-1}, 95 \%$ confidence interval $=1.0 \mathrm{I}-$ $1.08, p=0.015)$ and styloid process-internal carotid artery distance $\left(O R=0.77 \mathrm{~mm}^{-1}, 95 \%\right.$ confidence interval $=0.64$ $0.92, p=0.004)$.

Conclusion: Longer styloid process and shorter distance between styloid process and cervical internal carotid artery are associated with cervical internal carotid artery dissection.

\section{Keywords}

Internal carotid artery, cervical artery dissection, styloid process

Received: 14 April 2017; accepted: 5 August 2017

\section{Introduction}

Cervical artery dissection is one of the most frequent causes of ischemic stroke in young patients. ${ }^{1}$ In addition to some conditions with an established causative role in cervical artery dissection, such as monogenic connective tissue disorders and major trauma, pathophysiology is poorly understood and complex. ${ }^{2}$ Elongated styloid process (SP), usually defined as
'Neuroradiology Department, Hospital de Braga, Braga, Portuga ${ }^{2}$ Medical Imaging Department, Neuroradiology Functional Unit, Centro Hospitalar e Universitário de Coimbra, Coimbra, Portugal

${ }^{3}$ Neuroradiology Department, Centro Hospitalar de Vila Nova de Gaia/ Espinho, Espinho, Portugal

${ }^{4}$ Neurology Department, Centro Hospitalar e Universitário de Coimbra, Coimbra, Portugal

${ }^{5}$ Neurology Department, Hospital de Braga, Portugal

${ }^{6}$ Neurology Department, Centro Hospitalar de Vila Nova de Gaia / Espinho, Portugal

Corresponding author:

João Pinho. Neurology Department, Hospital de Braga, Sete Fontes, São Victor, Braga 47I0-243, Portugal.

Email: joao.pinho@hospitaldebraga.pt. 
$>30 \mathrm{~mm},{ }^{3}$ may present with Eagle syndrome as the carotid subtype ${ }^{4}$ and has been reported in patients with cervical internal carotid artery dissection (ICAD) even with no previous clinical manifestations. ${ }^{5}$ More recently, anatomical characteristics of SP have gained attention as possible predisposing factors for ICAD. ${ }^{6}$ This study aimed to replicate previous studies by characterizing SP length and SP distance to internal carotid artery (ICA) in patients with ICAD.

\section{Methods}

Retrospective, multicenter, case-control study of consecutive patients with cervical ICAD presenting with ischemic stroke, transient ischemic attack (TIA), or only local symptoms and signs, with available computed tomography angiography (CTA) performed in the first two weeks of diagnosis, diagnosed between January 2010 and September 2016. Age- and sexmatched controls were consecutive patients with ischemic stroke or TIA, caused by any etiology excluding ICAD, who performed CTA during the acute phase, selected from the outpatient clinic database of one of the participating departments. CTA images were individually reviewed by two independent observers for measurements of SP length and SP-ICA distance (ipsilateral and contralateral to dissection or left and right in controls). Observers were not blinded to dissection status. SP length was measured using oblique multiplanar reconstructions. SP-ICA distance in the axial plane, in the slice where the distance between the two structures was shorter, was measured between the edge of the SP and the ICA lumen center in controls or the center of the structure formed by the ICA lumen and the intramural hematoma in cases (Figure 1). Mean values of the two independent measurements were used for the analyses. We characterized the existence of cervical ICA tortuosity and kinking according to previously described criteria. ${ }^{7}$ Statistical analysis was performed using chi-square and independent student's

Figure I. Examples of measurements of styloid process length and styloid process-internal carotid artery distance in a control subject $(a, c)$ and in a case $(b, d)$.
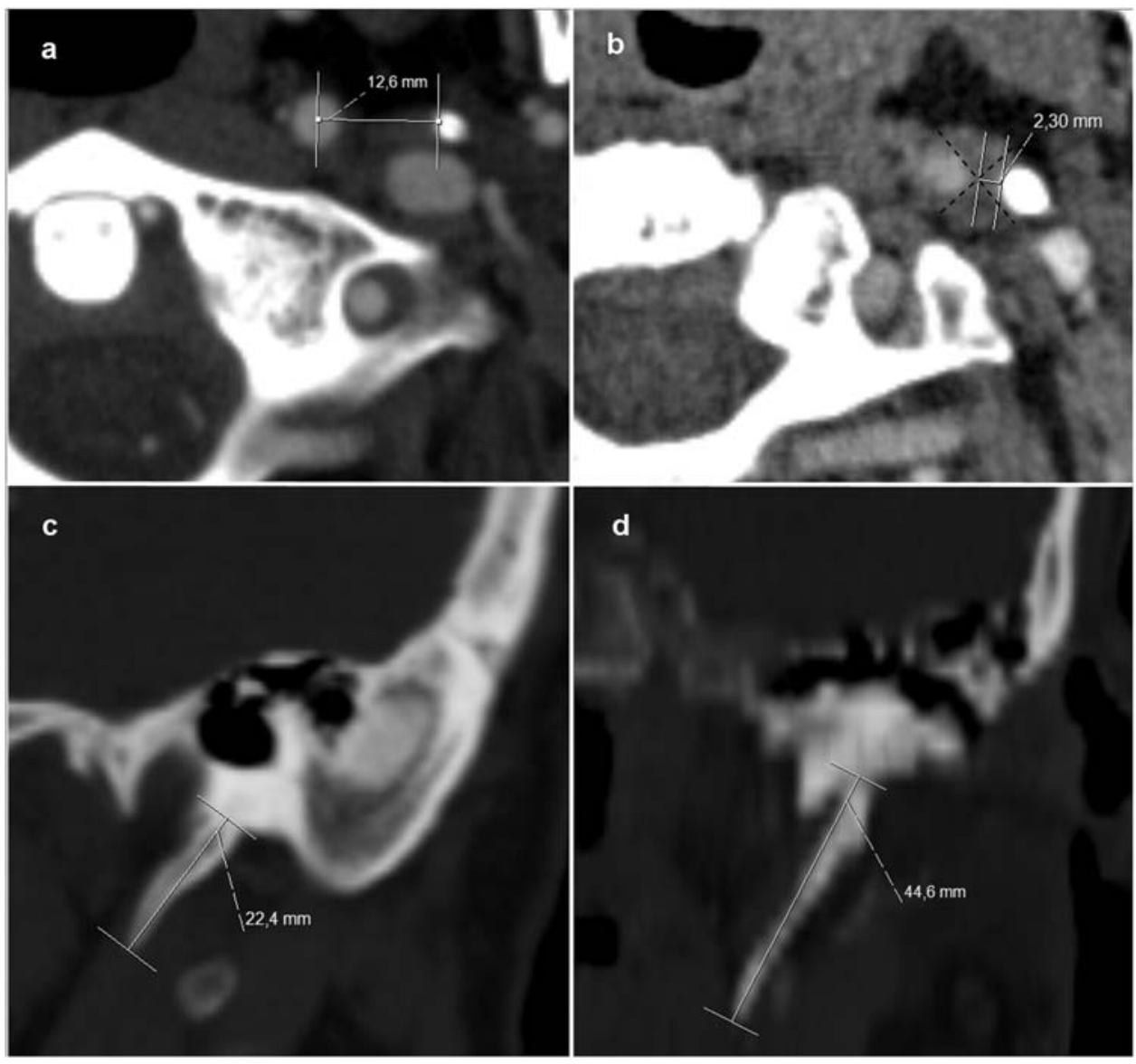
t-test for comparison of the two groups, univariate binomial logistic regression to calculate odds ratio $(\mathrm{OR})$ and $95 \%$ confidence interval $(95 \% \mathrm{CI})$ associated with SP length and SP-ICA distance, receiver operating characteristic (ROC) curves to calculate the area under the curve (AUC). Interobserver agreement was calculated using interclass correlation (ICC) for single measures. Statistical significance was defined as $\mathrm{p}<0.05$. IBM SPSS Statistics version 22 and MedCalc were used for the statistical analyses.

\section{Results}

Sixty-two cases, of which three had bilateral ICAD (65 dissected arteries), and 70 controls were included in the study. There were no differences in age, sex, or admission stroke severity between groups (Table 1). Among patients with ICAD, $14.5 \%$ presented with only local symptoms and signs (acute manifestations of ICAD), $79 \%$ presented with ischemic stroke, and $6.5 \%$ presented with TIA. Horner syndrome was present in $33.9 \%$. Major trauma as a cause of ICAD occurred in only one patient. No patient with ICAD was diagnosed with fibromuscular dysplasia. All included cases and controls performed CTA and 53 ICAD patients $(85.5 \%)$ additionally performed $\geq 1$ angiographic exam to support the diagnosis (Table 1). Among controls, the cause of ischemic stroke was identified in 48 patients, and 22 patients had cryptogenic stroke. Eagle's syndrome was present in one of the ICAD patients and in none of the controls. Frequency of cervical ICA tortuosity and kinking in the ICAD group was similar to the control group (Table 1). Nine patients with ICAD were treated with stenting, which was performed, in all cases, after diagnostic CTA.

Interobserver agreement was good for SP length (ICC coefficient $=0.89,95 \% \mathrm{CI}=0.86-0.91)$ and $\mathrm{SP}-$ ICA distance $(\mathrm{ICC}=0.76,95 \% \mathrm{CI}=0.70-0.80)$. Mean SP length ipsilateral to dissection was $35.8 \mathrm{~mm}( \pm 14.4)$, compared to left SP length of $30.4 \mathrm{~mm}( \pm 8.9)$ and right SP length of $30.3 \mathrm{~mm}( \pm 8.2)$ in controls $(\mathrm{p}=0.011$ and 0.008 , respectively) (Figure 2(a)). SP length $>30 \mathrm{~mm}$ ipsilateral to dissection occurred in $60.0 \%$ of patients, and SP length $>30 \mathrm{~mm}$ (either side) occurred in $41.4 \%$ of patients in the control group $(p=0.039)$. Mean SP length contralateral to dissection $(32.3 \mathrm{~mm}[ \pm 11.7])$ was not significantly different from SP length ipsilateral to dissection $(p=0.061)$. Overall, males had longer SP than females $(33.8 \pm 13.1 \mathrm{~mm}$ versus $29.7 \pm 6.5 \mathrm{~mm}$, $\mathrm{p}=0.018$ ), and there was no correlation between SP length and age. Mean SP-ICA distance ipsilateral to dissection was $6.3 \mathrm{~mm}( \pm 1.9)$, compared to left $\mathrm{SP}-$ ICA distance of $7.2 \mathrm{~mm}( \pm 2.1)$ and right SP-ICA distance of $7.0 \mathrm{~mm}( \pm 2.3)$ in controls $(\mathrm{p}=0.003$ and $\mathrm{p}=0.026$, respectively) (Figure 2(b)). Mean SP-ICA distance contralateral to dissection $(6.7 \mathrm{~mm}[ \pm 2.2])$ was not significantly different from SP-ICA distance ipsilateral to dissection $(p=0.160)$. Logistic regression revealed an OR for ICAD of $1.04(95 \% \mathrm{CI}=1.01-1.08$, $\mathrm{p}=0.015)$ for $1 \mathrm{~mm}$ increase in SP length, and an OR for ICAD of $0.77(95 \% \mathrm{CI}=0.64-0.92, \mathrm{p}=0.004)$ for $1 \mathrm{~mm}$ increase in SP-ICA distance. ROC curves analyses for ICAD diagnosis revealed AUC for SP length of $0.615(95 \% \mathrm{CI}=0.528-0.698)$ and AUC for SP-ICA distance of $0.645(95 \% \mathrm{CI}=0.558-0.725)$ (supplementary material).

\section{Discussion}

In this multicenter case-control study, increasing SP length and decreasing SP-ICA distance were associated with ICAD. Only three previous case-control studies systematically analyzed the morphological characteristics of SP in ICAD patients. In the first study, ${ }^{6}$ the authors found that carotid dissection was associated with longer SPs, with an OR per millimeter increase of 1.08 , similar to the result we found in our study. Additional anatomical characteristics such as SP angulation and SP-ICA proximity were found not to be associated with dissection. Methodological issues may limit comparison between studies, specifically in what concerns SP-ICA distance measurement. We adopted the method used in another study, which in addition to confirm longer SP ipsilateral to ICAD, also found that the distances between SP and dissected carotid artery were significantly shorter when compared to SP-ICA distances contralateral to dissection and in controls. ${ }^{8}$ Because intramural hematoma may distort ICA anatomy and change its position in relation to other fixed cervical structures, we believe that measurement of the distance between SP and the center of the structure formed by the hematoma and the arterial lumen describes a more reliable anatomical relation between these structures, even though it does not completely exclude the bias introduced by large position changes, such as those caused by pseudoaneurysms. Muthusami et al. ${ }^{9}$ also found a marginal association of longer SP length and shorter SP-ICA distance in ICAD patients when compared to controls; however, this study was conducted in a single center, included fewer cases, and used a different method to measure SP-ICA distance. ${ }^{9}$ In contrast to the first two studies, ${ }^{6,8}$ we found that the characteristics of the SP contralateral to ICAD were not significantly associated with dissection, which could explain the low frequency of bilateral ICAD. However, this association was only marginally nonsignificant, which could be related to the sample size.

Although the present study adds confirmatory evidence for the association of ICAD with SP length and its proximity to ICA, it is not able to confirm a causal 
Table I. Baseline characteristics of cases and controls

\begin{tabular}{|c|c|c|}
\hline & $\begin{array}{l}\text { Cervical internal } \\
\text { carotid artery } \\
\text { dissection }(n=62)\end{array}$ & Controls $(n=70)$ \\
\hline Age (years) & $50.6( \pm 1 \mathrm{I} .1)$ & $49.6( \pm 12.9)$ \\
\hline Male & $44(71.0)$ & $48(68.6)$ \\
\hline \multicolumn{3}{|l|}{ Clinical manifestations } \\
\hline Ischemic stroke & $49(79.0)$ & $66(94.3)$ \\
\hline TIA & $4(6.5)$ & $4(5.7)$ \\
\hline Only local signs/symptoms & $9(14.5)$ & - \\
\hline Horner syndrome & $21(33.9)$ & - \\
\hline \multicolumn{3}{|l|}{ Ischemic stroke/TIA etiology } \\
\hline Cardioembolism & - & $10(14.3)$ \\
\hline Large vessel disease & - & $19(27.1)$ \\
\hline Small vessel disease & - & $8(11.4)$ \\
\hline Cryptogenic & - & $22(31.4)$ \\
\hline Other causes & - & II (I5.7) \\
\hline Cervical artery dissection & $53(100)$ & - \\
\hline Admission NIHSS (if ischemic stroke) & $8.3( \pm 7.1)$ & $8.2( \pm 7.6)$ \\
\hline Major trauma & I ( 1.6$)$ & - \\
\hline Minor trauma & $2(3.2)$ & - \\
\hline \multicolumn{3}{|l|}{ Angiographic exams } \\
\hline Computed tomography angiography & $62(100)$ & $70(100)$ \\
\hline Cervical ultrasound & $38(6 \mid .3)$ & $19(27.1)^{*}$ \\
\hline Magnetic resonance angiography & $19(30.6)$ & $8(11.4)^{*}$ \\
\hline Digital subtraction angiography & $12(19.4)$ & $19(27.1)$ \\
\hline \multicolumn{3}{|c|}{ Anatomical characteristics of cervical internal carotid artery } \\
\hline Tortuosity & $15(24.2)$ & $23(32.9)$ \\
\hline Kinking & $9(14.5)$ & $10(14.3)$ \\
\hline
\end{tabular}

NIHSS: National Institutes of Health Stroke Scale; TIA: transient ischemic attack.

Values are presented as $\mathrm{n}(\%)$ or mean ( \pm standard deviation). $*_{\mathrm{p}}<0.05$.

role. Several issues argue against a possible contribution of SP anatomical characteristics to ICAD pathophysiology: because SP elongation is not rare and there is a significant variability of SP length in the general population, ${ }^{10}$ one would expect ICAD to be more frequent; higher dissection recurrences were to be expected $^{11}$ if there was a persistent anatomical contributor to dissection; SP elongation is bilateral in most cases and bilateral ICAD is not frequent. ${ }^{12}$ Despite this, many additional predisposing factors 
Figure 2. (a) Quartile distribution of styloid process length $(\mathrm{mm})$ in cases (ipsilateral and contralateral to dissection) and controls (left and right). (a) Quartile distribution of the distance between the styloid process and internal carotid artery ( $\mathrm{mm}$ ) in cases (ipsilateral and contralateral to dissection) and controls (left and right). P values refer to independent t-student tests for comparison of means (ns: non-significant).

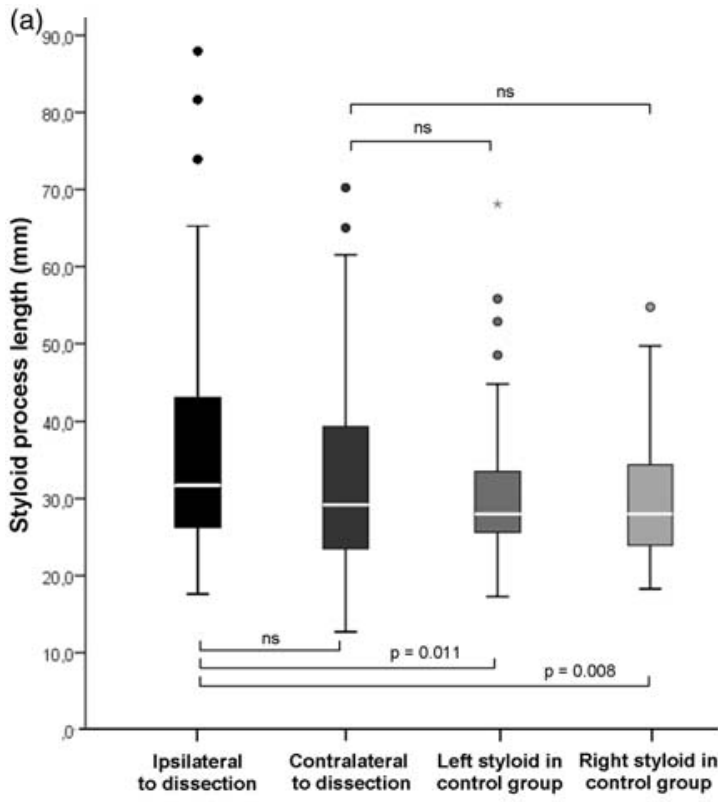

for cervical artery dissection have been recognized, and the occurrence of dissection in a specific time in a specific patient may result from the combination of several contributors. Head and neck movements or even mild cervical trauma in patients with elongated SP closer to ICA may lead to repeated arterial position displacement and mechanical trauma, predisposing to dissection. This study replicates the findings of the previous small case-control studies on this subject and has the strength of being a multicenter study with a high interobserver agreement. The choice of the control population was primarily based on the fact that the majority of patients who undergo CTA in our clinical practice have cerebrovascular disease. Even though there may exist some concerns regarding the validity of the control group, we reduced potential bias by only including controls who had complete etiological evaluation, with matching for age and sex, not including any control with ICA occlusion and, the fact that both groups had similar frequency of carotid artery anomalies such as kinking and significant tortuosity also increases comparability. Three-dimensional CTA reconstructions may be superior to conventional CTA because they can clarify the anatomy of SP and its relation to overlapping structures ${ }^{13}$; however, the use of oblique multiplanar reconstructions is useful for the selection of the optimal plane for SP measurement.

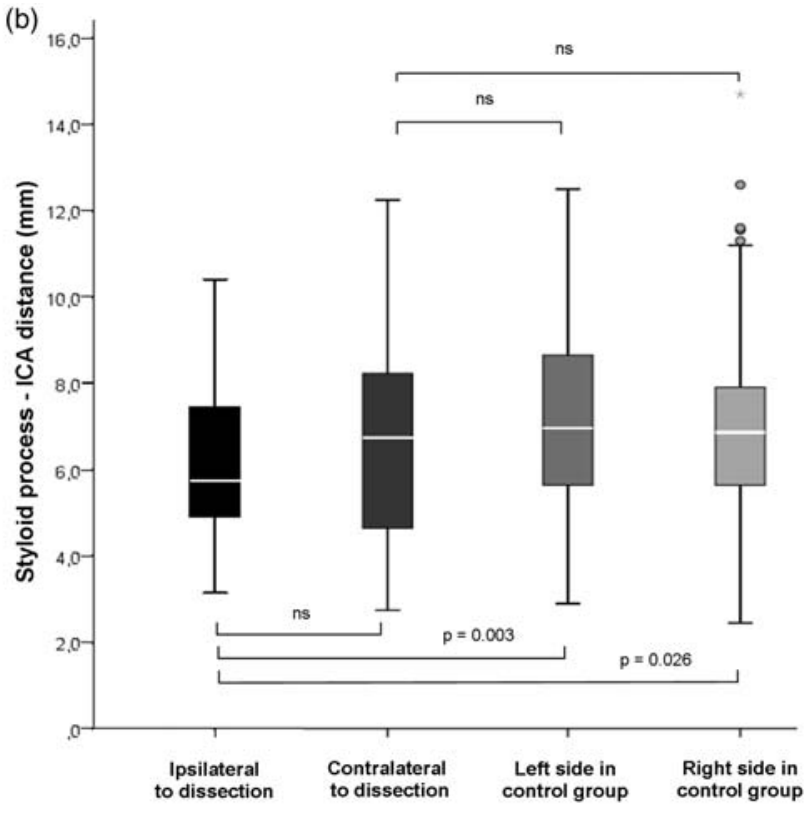

Additional limitations are related to the small number of cases, impossibility to perform SP measurements blinded to dissection status, and absent systematic information concerning head and neck movement precipitants.

\section{Conclusion}

Anatomical characteristics of the SP, namely SP length and SP-ICA distance, are associated with ICAD.

\section{Declaration of conflicting interests}

The author(s) declared no potential conflicts of interest with respect to the research, authorship, and/or publication of this article.

\section{Funding}

The author(s) received no financial support for the research, authorship, and/or publication of this article.

\section{References}

1. Ferro JM, Massaro AR and Mas JL. Aetiological diagnosis of ischaemic stroke in young adults. Lancet Neurol 2010; 9: 1085-1096.

2. Debette $\mathbf{S}$ and Leys D. Cervical-artery dissections: predisposing factors, diagnosis, and outcome. Lancet Neurol 2009; 8: 668-678. 
3. Ekici F, Tekbas G, Hamidi C, et al. The distribution of stylohyoid chain anatomic variations by age groups and gender: an analysis using MDCT. Eur Arch Otorhinolaryngol 2013; 270: 1715-1720.

4. Sveinsson O, Kostulas N and Herrman L. Internal carotid dissection caused by an elongated styloid process (Eagle syndrome). BMJ Case Rep. Epub ahead of print 11 June 2013.

5. Zuber M, Meder JF and Mas JL. Carotid artery dissection due to elongated styloid process. Neurology 1999; 53: $1886-1887$.

6. Raser JM, Mullen MT, Kasner SE, Cucchiara BL and Messé SR. Cervical artery dissection is associated with styloid process length. Neurology 2011; 77: 2061-2066.

7. Labeyrie PE, Braud F, Gakuba C, et al. Cervical artery tortuosity is associated with intracranial aneurysm. Int $J$ Stroke 2017; 12: 549-552.

8. Renard D, Azakri S, Arquizan C, Swinnen B, Labauge P and Thijs V. Styloid and hyoid bone proximity is a risk factor for cervical carotid artery dissection. Stroke 2013; 44: 2475-2479.
9. Muthusami P, Kesavadas C, Sylaja PN, Thomas B, Harsha KJ and Kapilamoorthy TR. Implicating the long styloid process in cervical carotid artery dissection. Neuroradiology 2013; 55: 861-867.

10. Piagkou M, Anagnostopoulou S, Kouladouros K and Piagkos G. Eagle's syndrome: a review of the literature. Clin Anat 2009; 22: 545-558.

11. Kremer C, Mosso M, Georgiadis D, et al. Carotid dissection with permanent and transient occlusion or severe stenosis. Neurology 2003; 60: 271-275.

12. Debette S, Grond-Ginsbach C, Bodenant M, et al. Differential features of carotid and vertebral artery dissections. The CADISP study. Neurology 2011; 77: 1174-1181.

13. Kent DT, Rath TJ and Snyderman C. Conventional and 3-dimensional computerized tomography in Eagle's syndrome, glossopharyngeal neuralgia, and asymptomatic controls. Otolaryngol Head Neck Surg 2015; 153: 41-47. 